\title{
Revisiting unitarity corrections for electromagnetic processes in collisions of relativistic nuclei
}

\author{
U.D. Jentschura ${ }^{1,2}$, K. Hencken ${ }^{3,4}$, V.G. Serbo ${ }^{2,5, a}$ \\ ${ }^{1}$ Max-Planck-Institut für Kernphysik, Postfach 1039 80, 69029 Heidelberg, Germany \\ ${ }^{2}$ Institut für Theoretische Physik, Philosophenweg 16, 69120 Heidelberg, Germany \\ ${ }^{3}$ Institut für Physik, Universität Basel, Klingelbergstr. 82, 4056 Basel, Switzerland \\ ${ }^{4}$ ABB Switzerland Ltd., Corporate Research, Segelhof 1K, 5405 Baden, Switzerland \\ ${ }^{5}$ Novosibirsk State University, Pirogova 2, 630090 Novosibirsk, Russia
}

Received: 25 July 2008 / Published online: 22 October 2008

(C) Springer-Verlag / Società Italiana di Fisica 2008

\begin{abstract}
Unitarity corrections to several electromagnetic processes in collisions of relativistic heavy nuclei are considered. They are due to the unitarity requirement for the $S$-matrix and correspond to the exchange of a light-by-light scattering block between colliding nuclei. We obtain improved results for the corrections to $e^{+} e^{-}$and $\mu^{+} \mu^{-}$pair production as well as new results for unitarity corrections to the production of photons via virtual Compton and virtual Delbrück scattering. These corrections can be numerically large; e.g., the $\mu^{+} \mu^{-}$pair production cross section is reduced by about $50 \%$ and nuclear bremsstrahlung by about $15 \div 20 \%$.
\end{abstract}

\section{Introduction}

The subject of this paper is the so-called unitarity corrections, which form a conceptually interesting class of corrections for quantum electrodynamic (QED) processes. The unitarity corrections come from the unitarity requirement for the $S$-matrix and are relevant for processes in which the lowest-order amplitude is large, i.e., in which a large number of photons and/or real electron-positron pairs are typically produced. One class of such processes is bremsstrahlung and lepton-pair production in ultra-relativistic heavy-ion collisions.

In order to put the current investigation into perspective, let us briefly recall that heavy-ion collisions definitely concern matter under extreme conditions. The impact parameters $\rho$ relevant for all cross sections under study in the cur-

a e-mail: serbo@math.nsc.ru rent paper are in the range of the electron Compton wavelength,

$\rho \sim \lambda_{e}=\frac{\hbar}{m c}=386 \mathrm{fm}$.

Now, the typical electric fields, as seen in the laboratory frame, generated by a particle moving at a speed characterized by a relativistic factor $\gamma$, are of the order of

$$
\begin{aligned}
\mathcal{E}(\gamma) & =\frac{Z e}{\hbar_{e}^{2}} \gamma=\frac{Z e \gamma m^{2} c^{2}}{\hbar^{2}} \\
& =\frac{m^{2} c^{3}}{\hbar e} \frac{Z e^{2}}{\hbar c} \gamma=\mathcal{E}_{\mathrm{cr}} Z \alpha \gamma,
\end{aligned}
$$

where $\mathcal{E}_{\text {cr }}$ is Schwinger's critical field strength. The quantity $Z \alpha \gamma$ assumes values in the range of $\sim 60$ for RHIC and $\sim 1800$ for the LHC, illustrating that the flash field accompanying the nuclei may well exceed the critical field by several orders of magnitude, for very small spatial regions and very small times.

In view of the huge pair-production and photoproduction cross sections encountered under these conditions, it is natural that the importance of unitarity corrections has been recognized for the first time within the production of electron-positron pairs in collisions of heavy nuclei (see [1] and the reviews in $[2,3]$ ). The unitarity correction for the one electron-positron pair production process,

$Z_{1}+Z_{2} \rightarrow Z_{1}+Z_{2}+e^{+} e^{-}$,

has been calculated in [4] and found to be about $3 \div 4 \%$. Estimates of unitarity corrections for the $\mu^{+} \mu^{-}$single-pair production process

$Z_{1}+Z_{2} \rightarrow Z_{1}+Z_{2}+\mu^{+} \mu^{-}$ 
Fig. 1 Feynman diagram for $e^{+} e^{-}$pair production in heavy-ion collision (first Born approximation). Bold lines denote nuclei, thin lines denote electrons
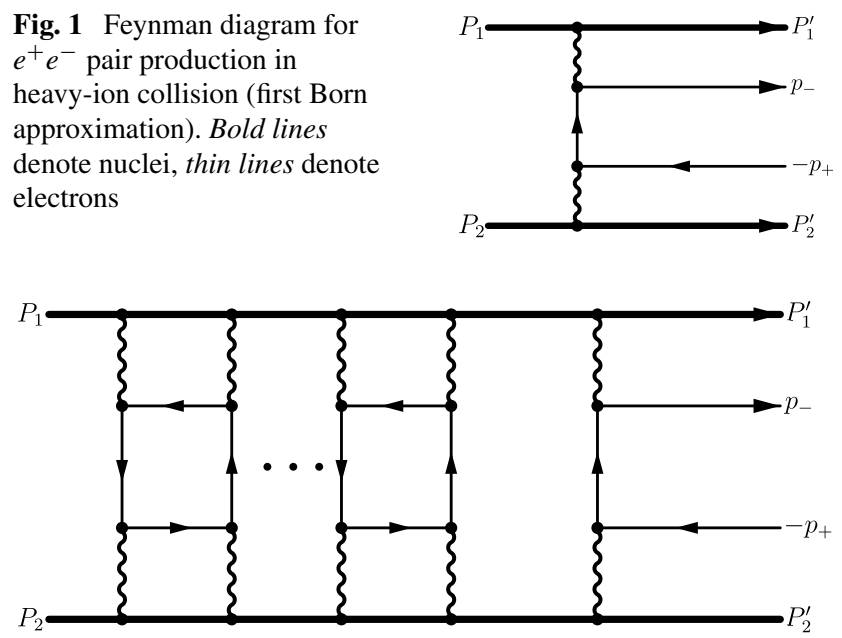

Fig. 2 Feynman diagram for the unitarity correction to $e^{+} e^{-}$pair production in heavy-ion collisions

have been obtained in [5]; in this case the unitarity correction is found to be large $(\sim 50 \%)$.

Let us consider these corrections conceptually using the process (3) as an example. In this case the lowest-order Feynman diagram is represented by Fig. 1, while the diagrams of the type depicted in Fig. 2 correspond to the unitarity correction. These diagrams include blocks of virtual light-by-light scattering via an electron loop, whose imaginary part corresponds to the production of electron-positron pairs by the Cutkosky rules.

For $Z_{1} Z_{2} \alpha \gg 1$ and $\gamma \gg 1$, it is possible to treat the nuclei as sources of an external field and to calculate the probability of $n$-pair production $P_{n}(\rho)$ in collisions of two nuclei at a fixed impact parameter $\rho$ [6]. The sum over $n$ of the probabilities $P_{n}(\rho)$ for $n$-pair production must be unity. The unitarity requirement is fulfilled by the Poisson distribution,

$P_{n}(\rho)=\frac{\bar{n}_{e}^{n}}{n !} \mathrm{e}^{-\bar{n}_{e}}$,

whose sum over $n$ gives 1 . Here $\bar{n}_{e} \equiv \bar{n}_{e}(\rho)$ is the averaged number of produced pairs at a given $\rho$, and the factor $\exp \left(-\bar{n}_{e}\right)$ is the vacuum-to-vacuum transition probability

$P_{0}(\rho)=\mathrm{e}^{-\bar{n}_{e}}=1-\sum_{n=1}^{\infty} P_{n}(\rho)$.

Roughly speaking, the probability for producing one pair, given in perturbation theory by $\bar{n}_{e}$, should be modified to read $\bar{n}_{e} \exp \left(-\bar{n}_{e}\right)$, and this correction is not small for an appreciable value of $\bar{n}_{e}$. This means that also the cross section for the one-pair production $\sigma_{1}$ should be multiplied by an appropriate factor $\exp \left(-\bar{n}_{e}\right)$ in the integral over the impact parameter, which corresponds to the following replacement:

$\sigma_{1}=\int \bar{n}_{e}(\rho) \mathrm{d}^{2} \rho \rightarrow \sigma_{1}+\sigma_{1}^{\text {unit }}=\int \bar{n}_{e}(\rho) \mathrm{e}^{-\bar{n}_{e}(\rho)} \mathrm{d}^{2} \rho$,
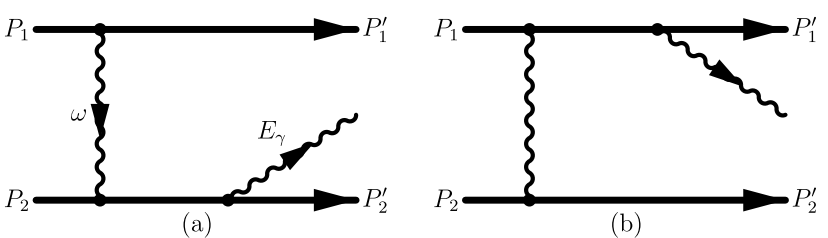

Fig. 3 Ordinary nuclear bremsstrahlung is the emission of a photon in a nuclear collision via a virtual Compton subprocess

where, finally,

$\sigma_{1}^{\text {unit }}=-\int \bar{n}_{e}(\rho)\left[1-\mathrm{e}^{-\bar{n}_{e}(\rho)}\right] \mathrm{d}^{2} \rho$

is the unitarity correction to the one-pair production cross section.

In $[4,5]$, rather rough approximations were used for the function $\bar{n}_{e}(\rho)$. Here, our intention is to use improved approximations for these functions. Thus, the aims of the present paper are (i) to revise the problem of the impactparameter dependent pair-production probability $\bar{n}_{e}(\rho)$, (ii) to update the unitarity corrections for the previously calculated processes (3) and (4), (iii) to calculate the unitarity correction for photon emission at nuclear collisions,

$Z_{1}+Z_{2} \rightarrow Z_{1}+Z_{2}+\gamma$.

In the latter case, the probability for the production of one and only one photon in a heavy-ion collision is modified by the necessity of suppressing the possibility of simultaneous production of electron-positron pairs. In the impactparameter representation, this implies that the cross section for photoproduction, $\sigma_{\gamma}$, should also be multiplied by the factor $\exp \left(-\bar{n}_{e}\right)$. This corresponds to the replacement

$$
\begin{aligned}
\sigma_{\gamma} & =\int P_{\gamma}(\rho) \mathrm{d}^{2} \rho \rightarrow \sigma_{\gamma}+\sigma_{\gamma}^{\text {unit }} \\
& =\int P_{\gamma}(\rho) \mathrm{e}^{-\bar{n}_{e}(\rho)} \mathrm{d}^{2} \rho,
\end{aligned}
$$

where $P_{\gamma}(\rho)$ is the probability to emit a photon in the collision of two nuclei at a given impact parameter $\rho$. Therefore, the unitarity correction in this case is given by the expression

$\sigma_{\gamma}^{\text {unit }}=-\int P_{\gamma}(\rho)\left[1-\mathrm{e}^{-\bar{n}_{e}(\rho)}\right] \mathrm{d}^{2} \rho$.

It should be mentioned that there are two different mechanisms for photon emission in nuclear collisions: ordinary bremsstrahlung via virtual Compton scattering (see Fig. 3) and the recently considered $[7,8]$ emission of a photon via virtual Delbrück scattering, as illustrated in Fig. 4.

Both of the above-mentioned corrections to $e^{+} e^{-}$pair production and to photoproduction correspond to the exchange of virtual light-by-light scattering interactions between the nuclei. Each block of light-by-light scattering 

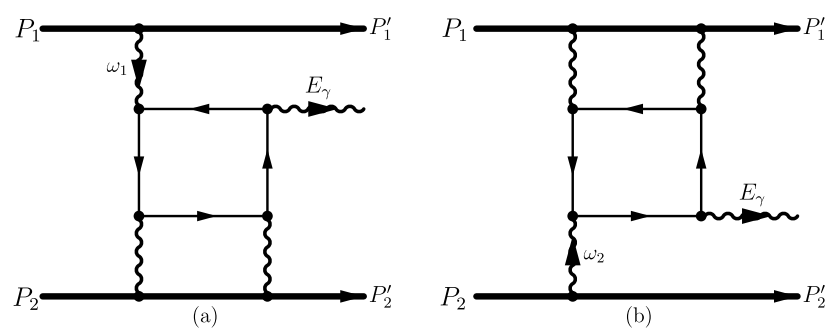

Fig. 4 Emission of a photon in a nuclear collision via virtual Delbrück scattering in the lowest order of QED

brings in an additional factor $\left(Z_{1} \alpha Z_{2} \alpha\right)^{2}$ in the amplitude of the corresponding process, and therefore such corrections can be omitted for the scattering of light ions, for muonnucleus or electron-nucleus scattering. We definitely need $Z$ to be large for the correction to be appreciable, and consequently this paper is focused on heavy-ion collisions.

We organize our paper as follows. In Sect. 2, we briefly recall relevant physical parameters for modern heavy-ion machines and discuss a new approximation for the function $\bar{n}_{e}(\rho)$. Section 3 is devoted to the actual calculation of the corrections. Specifically, we consider unitarity corrections to $e^{+} e^{-}$and $\mu^{+} \mu^{-}$pair production in Sects. 3.1 and 3.2, respectively, and we reserve the calculation of unitarity corrections to the production of photons via virtual Compton scattering to Sect. 3.3 and via virtual Delbrück scattering to Sect. 3.4. Finally, some conclusions are drawn in Sect. 4.

\section{Toward a revised representation of the impact-parameter dependent pair production probability}

Recently, electromagnetic processes in ultra-relativistic nuclear collisions have found strong and partially renewed interest in numerous papers (see the reviews $[2,3]$ and references therein). Of topical importance are the RHIC collider and the future $\mathrm{LHC} \mathrm{Pb}-\mathrm{Pb}$ option. It is therefore useful to recall the basic physical parameters of these colliders, namely the charge numbers of nuclei $Z_{1}=Z_{2} \equiv Z$ and their Lorentz factors $\gamma_{1}=\gamma_{2} \equiv \gamma$. These are given in Table 1 , which is cited here from [9]. Many of electromagnetic particle-production processes are of imminent importance for two reasons: they are either "dangerous," e.g. in terms of possible beam losses and background, or they are by contrast quite useful for monitoring some experiments at the RHIC and LHC colliders [10].

To fix the conventions used, we mention that natural units with $\hbar=c=1$ and with the fine-structure constant $\alpha \approx 1 / 137$ are used throughout the text, and we denote the electron and muon masses by $m$ and $\mu$, respectively.

As was mentioned in the introduction, the importance of unitarity corrections has been recognized for the first time at the process of electron-positron pair production be-
Table 1 Nuclear charge numbers $Z$ and relativistic $\gamma$ factors for modern heavy-ion machines

\begin{tabular}{lrr}
\hline Collider & $Z$ & \multicolumn{1}{c}{$\gamma$} \\
\hline RHIC, $\mathrm{Au}-\mathrm{Au}$ & 79 & 108 \\
LHC, $\mathrm{Pb}-\mathrm{Pb}$ & 82 & 3000 \\
\hline
\end{tabular}

cause a large number of real electron-positron pairs are typically produced in ultra-relativistic heavy-ion collisions. In the lowest QED order (Born approximation) this process is described by the Feynman diagram of Fig. 1. Let $\bar{n}_{e} \equiv \bar{n}_{e}(\rho)$ be the expected (average) number of pairs to be produced in the collision of two nuclei at a given impact parameter $\rho$. A closed form of the corresponding expression was obtained in $[11,12]$ although a complete and consistent interpretation of the expressions found was only given later in [13]. One issue is that $\bar{n}_{e}(\rho)$ derived in $[11,12]$ actually requires a further regularization, which was implemented in $[14,15]$.

As is evident from (8) and (11), the function $\bar{n}_{e}(\rho)$ is a very important quantity for the evaluation of unitarity corrections. However, the obtained closed form for $\bar{n}_{e}(\rho)$ is, in fact, a nine-fold integral and its calculation is very laborious. Therefore, a simpler approximate expression for $\bar{n}_{e}(\rho)$ is very desirable.

The properties of $\bar{n}_{e}(\rho)$ have been studied in detail in $[4,16]$. The corresponding functional form for identical heavy nuclei in the region $\rho<\gamma / m$ reads

$$
\begin{gathered}
\bar{n}_{e}(\rho, \gamma, Z)=(Z \alpha)^{4} F(x, Z)[L-G(x, Z)], \\
L=\ln \left(\gamma^{2}\right), x=m \rho .
\end{gathered}
$$

Here, $\gamma \gg 1$ is the usual relativistic factor (see Table 1). The analytical expressions for the functions $F(x, Z)$ and $G(x, Z)$ have been obtained in $[4,16]$ only for large values of the impact parameters, $1 \ll x=m \rho<\gamma$ (see the Appendix):

$F(x, Z)=\frac{56}{9 \pi^{2}} \frac{\ln x-f(Z \alpha)}{x^{2}}$,

$F(x, Z) \cdot G(x, Z)=\frac{56}{9 \pi^{2}} \frac{(3 / 2) \ln x-f(Z \alpha)}{x^{2}} \ln x$,

where

$f(Z \alpha)=(Z \alpha)^{2} \sum_{n=1}^{\infty} \frac{1}{n\left[n^{2}+(Z \alpha)^{2}\right]}$

is the well-known Bethe-Maximon function (in particular, $f(79 \alpha)=0.3129, f(82 \alpha)=0.3318)$. On the other hand, for calculations related to unitarity corrections we should know the functions $F(x, Z)$ and $G(x, Z)$ in the range $x \sim 1$.

In [16] a closed-form expression for the function $F(x, Z)$ at intermediate impact parameters in the form of a fivefold integral has been given. Tables provided in [16] give 
Table 2 The functions $A(x)$ and $B(x)$ as given in (16), calculated using the approach outlined previously in [17]

\begin{tabular}{lllllllll}
\hline$x$ & $A(x)$ & $B(x)$ & $x$ & $A(x)$ & $B(x)$ & $x$ & $A(x)$ & $B(x)$ \\
\hline 0.01 & 3.537 & 8.200 & 0.40 & 1.028 & 2.910 & 4.50 & 0.07273 \\
0.02 & 3.082 & 7.434 & 0.50 & 0.8879 & 2.573 & 5.00 & 0.06191 \\
0.03 & 2.805 & 6.927 & 0.60 & 0.7767 & 2.285 & 5.50 & 0.05327 & 0.3355 \\
0.04 & 2.611 & 6.535 & 0.70 & 0.6895 & 2.085 & 6.00 & 0.04635 \\
0.05 & 2.460 & 6.199 & 0.90 & 0.5557 & 1.747 & 6.50 & 0.04072 \\
0.06 & 2.331 & 5.892 & 1.00 & 0.5039 & 1.614 & 7.00 & 0.03606 \\
0.07 & 2.218 & 5.613 & 1.50 & 0.3293 & 1.142 & 7.50 & 0.03217 \\
0.08 & 2.122 & 5.366 & 2.00 & 0.2323 & 0.8581 & 8.00 & 0.02887 \\
0.09 & 2.037 & 5.139 & 2.50 & 0.1725 & 0.6716 & 8.50 & 0.1903 \\
0.10 & 1.962 & 4.949 & 3.00 & 0.1331 & 0.5431 & 9.00 & 0.1730 \\
0.20 & 1.486 & 3.900 & 3.50 & 0.1066 & 0.4560 & 9.50 & 0.02604 \\
0.30 & 1.217 & 3.345 & 4.00 & 0.08717 & 0.3879 & 10.0 & 0.1449 \\
\hline
\end{tabular}

a very clear numerical picture of the function $F(x, Z)$ for $x=0.01 \div 100$ and several important values of $Z$. For the function $G(x, Z)$, the approximation

$G(x, Z) \approx 1.5 \ln (x+1)+1.2$

(independent of $Z$ ) has been given in [16] as a rough indicator of the non-logarithmic (in $\gamma$ ) term in (12).

We improve this approximation by using the results from a first Born approximation, and a different parameterization has been employed altogether, namely

$\bar{n}_{e}(\rho)=(Z \alpha)^{4}[A(x) L-B(x)]$

(for a list of numerical values, see Table 2). There is an obvious connection between the above two sets of functions in the limit of low nuclear charge numbers,

$F(x, Z \rightarrow 0)=A(x), \quad G(x, Z \rightarrow 0)=\frac{B(x)}{A(x)}$.

First of all, it is reassuring to verify, based on the numerical data presented in [16, 17], that the equality $F(x, 0)=A(x)$ is valid in the phenomenologically important interval $x=$ $0.01 \div 10$ with an accuracy better than $5 \%$.

One can now take the data for $F(x, 0)$ as given in [16] and use a least-squares method in order to fit $G(x, 0)$ to numerical data in Table 2, assuming the functional form (16). A least-squares fit assuming the dependence

$G(x, 0)=1.5 \ln (x+a)+b$

gives as the best estimates $a=1.4$ and $b=1.9$, where the prefactor of the logarithm is fixed by the ratio of the two quantities discussed in (13). The approximation thus obtained differs from $B(x) / A(x)$ in the phenomenologically most important interval $x=0.02 \div 5$ by less than $2 \%$.
Based on the deviation of $F(x, Z=0)$ from $F(x, Z)$ by no more than $25 \%$ due to Coulomb corrections for heavy nuclei [16], we expect that the same deviation is valid in a comparison of $G(x, Z=0)$ and $G(x, Z)$. Taking into account that the function $G(x, Z)$ is the subleading term of the relative order of $1 / L$, we can conclude that the approximate expression

$$
\begin{gathered}
\bar{n}_{e}(\rho) \approx(Z \alpha)^{4} F(x, Z)[L-1.5 \ln (x+1.4)-1.9], \\
L=\ln \left(\gamma^{2}\right), x=m \rho,
\end{gathered}
$$

which involves the function $F(x, Z)$ from [16], has an accuracy of the order of $5 \%$. In the calculations reported below, we use this very expression.

\section{Calculation of unitarity corrections}

3.1 Unitarity corrections for $e^{+} e^{-}$pair production

Unitarity corrections for the process (3) have been considered in $[4,18]$. Based on (8), we find that the unitarity correction is

$\sigma_{e^{+} e^{-}}^{\text {unit }}=-\int_{\rho_{\min }}^{\rho_{\max }}\left[1-\mathrm{e}^{-\bar{n}_{e}(\rho)}\right] \bar{n}_{e}(\rho) \mathrm{d}^{2} \rho$,

where the integration limits (minimum and maximum impact parameters) are to be specified below. Since we are interested in so-called "silent events" without any "touching" of the nuclei, the physically allowed minimal value of the impact parameter is

$\rho_{\min }=2 R$,

where $R$ is the nuclear radius. A priori, the upper limit is $\rho_{\max }=\infty$, but due to fast convergence of the integral we can use as well $\rho_{\max }=100 / m$ for a quantitative estimate 
of the unitarity correction. Indeed, for large $\rho$, the asymptotics (13) allow for an expansion of the exponential for the vacuum persistence amplitude in (10) and in analogous expressions used for the unitarity corrections in this article, and thus there is a sufficiently large negative power of $\rho$ characterizing all integrands for large $\rho$; we can thus neglect excessively large impact parameters in the evaluation of all unitarity corrections. As a result, the integration region in the variable $x$ can be safely chosen as

$x_{0} \leq x=m \rho \leq x_{\max }=100, \quad x_{0}=m \rho_{\min }=2 m R$.

Here, $m$ is the electron mass. Using the relevant physical parameters, we find $x_{0}=0.0361$ for $\mathrm{Au}, x_{0}=0.0368$ for $\mathrm{Pb}$, $x_{0}=0.0385$ for $\mathrm{U}$ and $x_{0}=0.0213$ for Ca. Finally, the relative magnitude of the unitarity corrections for the considered $e^{+} e^{-}$pair-production process $(3)$ is

$\delta_{e^{+} e^{-}}=\frac{\sigma_{e^{+} e^{-}}^{\text {unit }}}{\sigma_{\text {Born }}}$

where the known Born cross section reads [19, 20]

$\sigma_{\text {Born }}=\frac{28}{27 \pi} \sigma_{0}\left[L^{3}-2.198 L^{2}+3.821 L-1.632\right]$,

with

$\sigma_{0}=\frac{\left(Z_{1} \alpha Z_{2} \alpha\right)^{2}}{m^{2}}, \quad L=\ln \left(\gamma_{1} \gamma_{2}\right)$.

For light nuclei with a non-excessive nuclear charge number $\left[(Z \alpha)^{4} L \ll 1\right]$, it is possible to calculate in the Born approximation the following integrals:

$C_{n}=\frac{2 \pi}{n !} \int_{x_{0}}^{x_{\max }} F^{n}(x) x \mathrm{~d} x, \quad n=2,3,4$,

$D_{2}=2 \pi \int_{x_{0}}^{x_{\max }} F^{2}(x) G(x) x \mathrm{~d} x$,

$E_{2}=\frac{2 \pi}{2 !} \int_{x_{0}}^{x_{\max }} F^{2}(x) G^{2}(x) x \mathrm{~d} x$,

where $F(x) \equiv F(x, Z=0)$ and $G(x) \equiv G(x, Z=0)$. They can be used to calculate, for light nuclei:

- The unitarity correction for light nuclei,

$$
\sigma_{e^{+} e^{-}}^{\text {unit }}=-2 \frac{(Z \alpha)^{8}}{m^{2}}\left(C_{2} L^{2}-D_{2} L+E_{2}\right) \text {. }
$$

- The total cross section for the production of two $e^{+} e^{-}$ pairs in collisions of light nuclei,

$\sigma_{2}=\frac{(Z \alpha)^{8}}{m^{2}}\left(C_{2} L^{2}-D_{2} L+E_{2}\right)$.

and
- The leading logarithmic asymptotics for the total cross section $\sigma_{n}$ for $n$-pair production with $n>2$,

$\sigma_{n}=\frac{(Z \alpha)^{4 n}}{m^{2}} C_{n} L^{n}$

Using the parameterization (19), we obtain the following numerical results for the coefficients listed in (26), which enter the formulas listed in (27a), (27b), and (27c) for various unitarity corrections and cross sections (in all integrals we used the integration region (22) with $x_{0}=0.0213$, which is the value obtained for a typical nucleus of low charge number, namely $\mathrm{Ca}$ ):

$$
\begin{array}{ll}
C_{2}=2.21, & C_{3}=0.443, \quad C_{4}=0.119, \\
D_{2}=15.5, & E_{2}=28.9 .
\end{array}
$$

The results from the previous investigations in [4] concern only those coefficients that can be defined exclusively in terms of $F(x)$. The previous results read $C_{2}=1.33, C_{3}=$ 0.264 , and $C_{4}=0.066$. These differ from the new results listed in (28), because in the previous investigation, a less accurate representation of $F(x)$ was used, which leads to discrepancies especially when higher powers of $F(x)$ enter the integrands as given in (26).

As an example, using the result (28), we found that the cross section for the production of two $e^{+} e^{-}$pairs for $\mathrm{Ca}-\mathrm{Ca}$ collisions at the LHC collider $(\gamma=3700)$ is

$\sigma_{2}=0.114$ barn

For heavier nuclei, one cannot use the Born approximation $F(x, Z) \approx F(x, Z=0) \equiv F(x)$ anymore. In this case, one has to resort to numerical data given in Table 1 of [16] for the heavy, collision systems $\mathrm{Au}-\mathrm{Au}$ and $\mathrm{Pb}-\mathrm{Pb}$ and employ the relativistic factors as given in Table 1. Indeed, unitarity corrections for the process $Z_{1} Z_{2} \rightarrow Z_{1} Z_{2} e^{+} e^{-}$have been considered in [4] and estimated to be $\delta_{e^{+}} e^{-}=-4.1 \%$ for $\mathrm{Au}-\mathrm{Au}$ at RHIC and $\delta_{e^{+} e^{-}}=-3.3 \%$ for $\mathrm{Pb}-\mathrm{Pb}$ at $\mathrm{LHC}$, where exactly the relativistic factors as given in Table 1 have been employed. We recall that the ratio $\delta_{e^{+} e^{-}}$has been defined in (23). Using (19), we are now in a position to present the new values

$\delta_{e^{+} e^{-}}=-5.0 \%$ for the RHIC,

$\delta_{e^{+} e^{-}}=-4.0 \%$ for the LHC,

which differ from those obtained in [4] by about $20 \%$.

3.2 Unitarity corrections for the $\mu^{+} \mu^{-}$pair production

Unitarity corrections for the process (4) have been roughly estimated in [5]. Based on the considerations leading to (8) 
and (11), we can immediately write down the corresponding formula:

$\sigma_{\mu^{+} \mu^{-}}^{\text {unit }}=-\int\left[1-\mathrm{e}^{-\bar{n}_{e}(\rho)}\right] P_{\mathrm{B}}(\rho) \mathrm{d}^{2} \rho$

where $P_{\mathrm{B}}(\rho)$ is the probability to produce a muon pair in collisions of two nuclei at a given impact parameter $\rho$ in the Born approximation (the Coulomb corrections to this probability, which correspond to multiphoton exchange of the produced $e^{+} e^{-}$with nuclei, are parametrically suppressed due to the large muon mass and can be neglected [5]).

For a simple calculation, we can use the expression for $P_{\mathrm{B}}(\rho)$ given in the leading logarithmic approximation (LLA) in [5]. It reads

$P_{\mathrm{B}}(\rho)=\frac{28}{9 \pi^{2}} \frac{\left(Z_{1} \alpha Z_{2} \alpha\right)^{2}}{(\mu \rho)^{2}} \Phi(\rho)$.

Depending on the value of $\rho$, the function $\Phi(\rho)$ assumes two different asymptotic forms, as shown in [5],

$\Phi(\rho)=\left[4 \ln \left(\frac{\gamma}{\mu \rho}\right)+\ln \left(\frac{\rho}{R}\right)\right] \ln \left(\frac{\rho}{R}\right) ; \quad R \ll \rho \leq \frac{\gamma}{\mu}$,

$\Phi(\rho)=\ln ^{2}\left(\frac{\gamma^{2}}{\mu^{2} \rho R}\right) ; \quad \frac{\gamma}{\mu} \leq \rho \ll \frac{\gamma^{2}}{\mu^{2} R}$.

This expression is valid for large values of $\ln (\rho / R)$, which is correct for LHC but not for RHIC. Therefore, below we consider the case of the LHC collider only.

Using formulae (31)-(33), we obtain

$\delta_{\mu^{+} \mu^{-}}=\frac{\sigma_{\mu^{+} \mu^{-}}^{\text {unit }}}{\sigma_{\mu^{+} \mu^{-}}}=-49 \%$ for the LHC,

where $\delta_{\mu^{+}} \mu^{-}$is of course defined as the relative magnitude of the unitarity correction in comparison to the Born cross section $\sigma_{\mu^{+} \mu^{-}}$, in analogy with (23).

The roughly tenfold increase of the unitarity correction (34) for muon-pair production in comparison to (30) for electron-positron pair production demands a qualitative explanation. Indeed, the importance of the unitarity correction is due to the enhanced contribution of the region of small impact parameters in the impact-parameter dependent muonpair production probability (32). Due to the prefactor $1 / \rho^{2}$ in (32), the unitarity correction is logarithmically enhanced as it involves an integration proportional to $\int \mathrm{d}^{2} \rho / \rho^{2}$ over the range of the impact parameter $2 R<\rho<1 / m$.

\subsection{Unitarity corrections for Compton-type photoproduction}

To a good approximation, tree-level photon emission by nuclear bremsstrahlung is described by the block Feynman diagrams of Fig. 3. Let the cross section $\mathrm{d} \sigma_{\mathrm{br}}^{a}$ and $\mathrm{d} \sigma_{\mathrm{br}}^{b}$ correspond to the diagrams of Figs. 3(a) and 3(b), respectively.
Roughly speaking, diagram (a) describes the emission of radiation by the Compton scattering of an equivalent photon, generated by nucleus 1 , off nucleus 2 , whereas for diagram (b), the situation is reversed. The cross section for photoproduction by nuclear bremsstrahlung then is obtained as the sum

$\mathrm{d} \sigma_{\mathrm{br}}=\mathrm{d} \sigma_{\mathrm{br}}^{a}+\mathrm{d} \sigma_{\mathrm{br}}^{b}$

because the interference term is small and can safely be neglected.

In the LLA, the cross section $\mathrm{d} \sigma_{\text {br }}^{a}$ can be calculated using the equivalent-photon approximation, in which it is expressed as follows:

$\mathrm{d} \sigma_{\mathrm{br}}^{a}=\mathrm{d} n_{1} \mathrm{~d} \sigma_{\mathrm{C}}\left(\omega, E_{\gamma}, E_{2}, Z_{2}\right)$.

Here, $\mathrm{d} n_{1}$ is the number of equivalent photons emitted by nucleus 1 in the energy interval $\mathrm{d} \omega$ and the impactparameter range $\mathrm{d}^{2} \rho$, and $\mathrm{d} \sigma_{\mathrm{C}}\left(\omega, E_{\gamma}, E_{2}, Z_{2}\right)$ is the differential cross section for the Compton scattering off nucleus 2 , for an energy $E_{\gamma}$ of the emitted photon, and an energy $E_{2}$ of the second nucleus of charge number $Z_{2}$ and mass $M_{2}$. The number of equivalent photons reads

$\mathrm{d} n_{1}=\frac{Z_{1}^{2} \alpha}{\pi^{2}} \frac{\mathrm{d} \omega}{\omega} \frac{\mathrm{d}^{2} \rho}{\rho^{2}}$,

with the integration region

$\omega_{\min } \leq \omega \lesssim \frac{\gamma_{1}}{\rho}, \quad 2 R \lesssim \rho \lesssim \rho_{\max }=\frac{\gamma_{1}}{\omega_{\min }}$

and

$\omega_{\min }=\frac{E_{\gamma}}{4 \gamma_{2}^{2}\left(1-x_{\gamma}\right)}$.

For the Compton cross section, we can use the following well-known expression, which is valid for a nucleus approximated by a charged point particle. This approach gives a good approximation at least in the region of not too energetic photons, where the nuclear structure can safely be neglected, and it reads

$$
\begin{aligned}
& \mathrm{d} \sigma_{\mathrm{C}}\left(\omega, E_{2}, E_{\gamma}, Z_{2}\right) \\
& \quad=4 \pi \frac{Z_{2}^{4} \alpha^{2}}{M_{2}^{2}}\left[\left(1-x_{\gamma}\right)\left(y-2 y^{2}+2 y^{3}\right)+\frac{12}{y x_{\gamma}^{2}}\right] \frac{\mathrm{d} E_{\gamma}}{E_{\gamma}},
\end{aligned}
$$

where

$x_{\gamma}=\frac{E_{\gamma}}{E_{2}}, \quad y=\frac{\omega_{\min }}{\omega}$. 
Then we integrate (36) over $\omega$ and write the result in the form

$\mathrm{d} \sigma_{\mathrm{br}}^{a}=\mathrm{d} P_{a}(\rho) \mathrm{d}^{2} \rho$,

where the differential probability $\mathrm{d} P_{a}(\rho)$ assumes the form

$\mathrm{d} P_{a}(\rho)=\frac{Z_{1}^{2} \alpha}{\pi^{2}} \frac{\sigma_{\mathrm{T}}\left(Z_{2}\right)}{\rho^{2}}\left(1-x_{\gamma}+\frac{3}{4} x_{\gamma}^{2}\right) \frac{\mathrm{d} E_{\gamma}}{E_{\gamma}}$,

with the Thomson cross section

$\sigma_{\mathrm{T}}\left(Z_{2}\right)=\frac{8 \pi}{3} \frac{Z_{2}^{4} \alpha^{2}}{M_{2}^{2}}$.

Formally the probability in (43) is divergent if integrated over all photon energies. However, using reasonable upper and lower bounds for $E_{\gamma}$, the probability remains small even at the minimal impact parameter.

According to the parameter region relevant for the equivalent-photon approximation (37), this expression is valid in the dominant region $2 R \leq \rho \lesssim \gamma_{1} / \omega_{\min }$. Integrating (42) over this region, we obtain

$\mathrm{d} \sigma_{\mathrm{br}}^{a}=2 \frac{Z_{1}^{2} \alpha}{\pi} \sigma_{\mathrm{T}}\left(Z_{2}\right)\left(1-x_{\gamma}+\frac{3}{4} x_{\gamma}^{2}\right) L_{\gamma} \frac{\mathrm{d} E_{\gamma}}{E_{\gamma}}$,

where

$L_{\gamma}=\ln \left(\frac{\rho_{\max }}{2 R}\right)=\ln \left(\frac{2 \gamma_{1} \gamma_{2}^{2}\left(1-x_{\gamma}\right)}{R E_{\gamma}}\right)$.

Now the unitarity correction $\delta_{\gamma}$, expressed as a fraction of the complete nuclear bremsstrahlung cross section, can be obtained by considering diagram (a) alone,

$\delta_{a}=\frac{\mathrm{d} \sigma_{\mathrm{unit}}^{a}}{\mathrm{~d} \sigma_{\mathrm{br}}^{a}}$,

and it can be calculated using

$\mathrm{d} \sigma_{\text {unit }}^{a}=-\int \mathrm{d} P_{a}(\rho)\left[1-\mathrm{e}^{-\bar{n}_{e}(\rho)}\right] \mathrm{d}^{2} \rho$.

The main (logarithmically enhanced) contribution to $\mathrm{d} \sigma_{\text {unit }}^{a}$ is given by the impact-parameter region $2 R \leq \rho \lesssim m^{-1}$, and therefore, a simple estimate can be given as

$\delta_{\gamma} \sim-(Z \alpha)^{4} \frac{L}{L_{\gamma}} \ln \left(\frac{1}{2 R m}\right)$.

A more accurate calculation is based on the direct integration of the vacuum persistence amplitude against pair production that involves the number of produced electronpositron pairs according to (12) and reads

$\delta_{\gamma}=-\frac{1}{L_{\gamma}} \int_{2 R}^{100 / m}\left[1-\mathrm{e}^{-\bar{n}_{e}(\rho)}\right] \frac{\mathrm{d} \rho}{\rho}$, where the convergence of the integral is assured by the asymptotics given in (13) and the upper limit of $100 / m$ for $\rho$ could have been replaced by $\infty$. An evaluation based on (50) gives the following result for $E_{\gamma}=1 \mathrm{GeV}$ :

$\delta_{\gamma}=-19 \%$ for the RHIC,

$\delta_{\gamma}=-15 \%$ for the LHC.

\subsection{Unitarity correction for Delbrück-type photoproduction}

Photon emission in heavy-ion collisions via virtual Delbrück scattering has recently been considered in [7,8], where a surprisingly large cross sections was found for this case:

$\sigma_{\gamma \mathrm{D}}=14$ barn for the RHIC,

$\sigma_{\gamma \mathrm{D}}=50$ barn for the LHC.

The main contribution to these cross sections comes from the photon-energy region

$m \ll E_{\gamma} \ll \gamma m$.

Below, we estimate the unitarity correction for the process discussed.

The probability $P_{\gamma \mathrm{D}}(\rho)$ entering the cross section (11) for photoproduction via virtual Delbrück scattering can easily be obtained as a function of the impact parameter $\rho$ in the dominant range $1 \ll m \rho \ll \gamma^{2}$, but the unitarity correction is mainly given by integration in the region $m \rho \sim 1$. Therefore, while we estimate the unitarity correction here, we stress that a more accurate calculation would require a direct evaluation of the Delbrück-type photoproduction probability $P_{\gamma \mathrm{D}}(\rho)$ in the range $m \rho \sim 1$, which was beyond our scope.

For the sake of simplicity, we consider here only the symmetric case $\gamma=\gamma_{1}=\gamma_{2}$ with identical nuclei $Z=Z_{1}=Z_{2}$. The cross section $\sigma_{\gamma \mathrm{D}}$ can be expressed by the integration of $P_{\gamma \mathrm{D}}(\rho)$ over the impact parameter via the relation

$\sigma_{\gamma \mathrm{D}}=\int P_{\gamma \mathrm{D}}(\rho) \mathrm{d}^{2} \rho$.

In LLA we can use the differential cross section in the same form as in (36):

$\mathrm{d} \sigma_{\gamma \mathrm{D}}=2 \mathrm{~d} n_{\gamma} \sigma_{\mathrm{D}}\left(Z_{2}\right)$,

where $\sigma_{\mathrm{D}}(Z)$ is a high-energy limit of the Delbrück scattering cross section defined according to (7) of [8], and

$\mathrm{d} n_{\gamma}=\frac{Z^{2} \alpha}{\pi^{2}} \frac{\mathrm{d} \omega}{\omega} \frac{\mathrm{d}^{2} \rho}{\rho^{2}}$

is the number of the equivalent photons. The factor 2 on the right-hand side of $\mathrm{d} n_{\gamma}$ takes into account two possibilities, corresponding two diagrams in Fig. 4. This expression 
is valid in a parameter range satisfying the two conditions $m / \gamma \lesssim \omega \lesssim \gamma / \rho$ and $1 / m \lesssim \rho \lesssim \gamma^{2} / m$. After integration of (56) over $\omega$, we obtain the probability $P_{\gamma \mathrm{D}}(\rho)$ in the form

$P_{\gamma \mathrm{D}}(\rho)=\frac{2 Z^{2} \alpha}{\pi^{2}} \frac{\sigma_{\mathrm{D}}(Z)}{\rho^{2}} \ln \left(\frac{\gamma^{2}}{m \rho}\right)$.

Under the restrictions for which the approximation made in (56) remains valid, the expression (57) is applicable in the dominant region

$1 \ll m \rho \ll \gamma^{2}$,

where again the probability is small. We rewrite (11) in the form

$\sigma_{\gamma \mathrm{D}}^{\text {unit }}=-\int P_{\gamma \mathrm{D}}(\rho)\left[1-\mathrm{e}^{-\bar{n}_{e}(\rho)}\right] \mathrm{d}^{2} \rho$,

and now we can use this formula in order to estimate the relative magnitude of the unitarity correction. The function $\bar{n}_{e}(\rho)$ is of the order of unity at $\rho \sim 1 / m$ and given by the expression

$\bar{n}_{e}(\rho) \approx 0.5(Z \alpha)^{4} L \quad(\rho \approx 1 / m)$,

but drops very quickly at larger impact parameters, with an asymptotic behavior of $\bar{n}_{e}(\rho) \propto 1 / \rho^{2}$. Since the function $P_{\gamma \mathrm{D}}(\rho)$ also drops at large impact parameters (see (57)), the main contribution to $\sigma_{\gamma \mathrm{D}}^{\text {unit }}$ comes from the region $\rho \sim 1 / \mathrm{m}$, and we can estimate the integral (59) as follows:

$$
\begin{aligned}
\sigma_{\gamma \mathrm{D}}^{\text {unit }} & \sim-\int P_{\gamma \mathrm{D}}(\rho) \bar{n}_{e}(\rho) \mathrm{d}^{2} \rho \\
& \sim-P_{\gamma \mathrm{D}}(1 / m) \bar{n}_{e}(1 / m) \frac{\pi}{m^{2}} .
\end{aligned}
$$

Taking into account the result (57), we obtain the estimate

$P_{\gamma \mathrm{D}}(\rho) \sim \frac{2 Z^{2} \alpha}{\pi^{2}} \sigma_{\mathrm{D}}(Z) m^{2} L \quad$ at $\rho \sim 1 / m$.

The relative magnitude of the unitarity correction thus is

$\delta_{\gamma \mathrm{D}}=\frac{\sigma_{\gamma \mathrm{D}}^{\mathrm{unit}}}{\sigma_{\gamma \mathrm{D}}} \sim-0.5(Z \alpha)^{4}$.

For the nuclear collisions at modern heavy-ion machines with parameters as listed in Table 1, one can estimate unitarity corrections to the photon emission to be on the level of $-5 \%$. Let us emphasize that the form of this correction (63) is different from that for the lepton-pair production discussed in Sects. 3.1 and 3.2.

\section{Conclusions}

In this article, we have considered unitarity corrections for $e^{+} e^{-}$and $\mu^{+} \mu^{-}$production in heavy-ion collisions, and for the production of photons by nuclear bremsstrahlung and by virtual Delbrück scattering. The main results of the current investigation can be found in (27a) for the unitarity correction to $e^{+} e^{-}$production for collisions of light nuclei, in (30) for the same process in heavy-ion collisions in modern colliders with parameters as given details for in Table 1 , for $\mu^{+} \mu^{-}$collisions in modern colliders (see (34)), where the unitarity correction is numerically large, and in (51) and (63) for the unitarity correction to photoproduction in heavy-ion machines, with allowance for both the ordinary nuclear bremsstrahlung and the virtual Delbrück scattering process.

Our results as presented for electron-positron pair production in (27a) and (30) are based on a refined treatment of the vacuum persistence amplitude against multipair production implied by (19), and they represent an update of results previously presented in [4] for the same corrections. For $\mu^{+} \mu^{-}$production, we update the results of [5]. For ordinary (Compton-type) and Delbrück-type photoproduction, the results for the unitarity corrections are obtained here for the first time to the best of our knowledge.

Finally, we notice that the estimates given here for the coefficients $C, D$ and $E$ in (28) also enter the total cross section for the production of two $e^{+} e^{-}$pairs in collisions of light nuclei (see (27b)) and the leading logarithmic asymptotics for the total cross section $\sigma_{n}$ for $n$-pair production with $n>2$ (see (27c)). From a phenomenological point of view, it is important to remark that all unitarity corrections reduce the one-photon or one-pair production cross sections, and that they can be numerically large (see (34) and (51)).

\section{Appendix}

In this appendix we briefly recall some details regarding the derivation of (13). The functions $F$ and $G$ from this equation enter the cross section of the process (3) as follows:

$\mathrm{d} \sigma_{1}=\bar{n}_{e}(\rho) \mathrm{d}^{2} \rho=\left(Z_{1} \alpha Z_{2} \alpha\right)^{2} F(x, Z)[L-G(x, Z)] \mathrm{d}^{2} \rho$.

Beyond the Born contribution, we should take into account, for this cross section, the so-called Coulomb corrections with a multiphoton exchange between the produced pair and the first or second nucleus:

$\mathrm{d} \sigma_{1}=\mathrm{d} \sigma_{\text {Born }}+\mathrm{d} \sigma_{\text {Coul }}$.

The Born contribution to the functions $F$ and $G$ was considered in detail in [4]. Thus, we only need to find the Coulomb corrections which enter (13) as items proportional to $f(Z \alpha)$. 
The Coulomb contribution to the total pair cross section was calculated in [21]; it can be presented in the form

$$
\begin{aligned}
\mathrm{d} \sigma_{\text {Coul }}= & \mathrm{d} n_{1} \sigma_{\text {Coul }}\left(\gamma_{1} Z_{2} \rightarrow e^{+} e^{-} Z_{2}\right) \\
& +\mathrm{d} n_{2} \sigma_{\text {Coul }}\left(\gamma_{2} Z_{1} \rightarrow e^{+} e^{-} Z_{1}\right),
\end{aligned}
$$

where

$\mathrm{d} n_{i}=\frac{Z_{i}^{2} \alpha}{\pi^{2}} \frac{\mathrm{d} \omega_{i}}{\omega_{i}} \frac{\mathrm{d}^{2} \rho}{\rho^{2}}$

is the number of the equivalent photons, produced by the $i$ th nucleus, and

$\sigma_{\text {Coul }}\left(\gamma Z \rightarrow e^{+} e^{-} Z\right)=-\frac{28}{9} \frac{Z^{2} \alpha^{3}}{m^{2}} f(Z \alpha)$

is the Coulomb correction to the total cross section of the photoproduction $\gamma Z \rightarrow e^{+} e^{-} Z$ taken from the well-known Bethe-Maximon formula. Integrating (A.4) over $\omega_{i}$ in the main region

$$
\frac{m}{\gamma_{2,1}} \lesssim \omega_{1,2} \lesssim \gamma_{1,2} m
$$

we find

$$
\begin{aligned}
\mathrm{d} \sigma_{\text {Coul }} & \\
= & -\frac{28}{9 \pi^{2}} \frac{\left(Z_{1} \alpha Z_{2} \alpha\right)^{2}}{(m \rho)^{2}}\left[f\left(Z_{1} \alpha\right)+f\left(Z_{2} \alpha\right)\right] \\
& \times \ln \left(\frac{\gamma_{1} \gamma_{2}}{m \rho}\right) \mathrm{d}^{2} \rho .
\end{aligned}
$$

Comparing this expression with (A.1) and (A.2) and assuming $Z_{1}=Z_{2}=Z$, we obtain both terms proportional to $f(Z \alpha)$ in (13).
Acknowledgements We are grateful to G. Baur, V. Fadin, I.F. Ginzburg, A. Milstein, N. Nikolaev and D. Trautmann for useful discussions. V.G.S. acknowledges the warm hospitality of the Institute of Theoretical Physics of Heidelberg University and support by the Gesellschaft für Schwerionenforschung (GSI Darmstadt, contract HDJENT). This work is partially supported by the Russian Foundation for Basic Research (code 06-02-16064) and by the Fond of Russian Scientific Schools (code 1027.2008.2). U.D.J. acknowledges support by the Deutsche Forschungsgemeinschaft (Heisenberg program).

\section{References}

1. G. Baur, Phys. Rev. A 42, 5736 (1990)

2. G. Baur, K. Hencken, D. Trautmann, S. Sadovsky, Y. Kharlov, Phys. Rep. 364, 359 (2002)

3. G. Baur, K. Hencken, D. Trautmann, Phys. Rep. 453, 1 (2007)

4. R.N. Lee, A.I. Milstein, V.G. Serbo, Phys. Rev. A 65, 022102 (2002)

5. K. Hencken, E.A. Kuraev, V.G. Serbo, Phys. Rev. C 75, 034903 (2007)

6. G. Baur, K. Hencken, A. Aste, D. Trautmann, S.R. Klein, Nucl. Phys. A 729, 787 (2003)

7. I.F. Ginzburg, U.D. Jentschura, V.G. Serbo, Phys. Lett. B 658, 125 (2008)

8. I.F. Ginzburg, U.D. Jentschura, V.G. Serbo, Eur. Phys. J. C 54, 267 (2008)

9. W.-M. Yao et al., J. Phys. G 33, 1 (2006)

10. A.J. Baltz et al., Phys. Rep. 458, 1 (2008)

11. B. Segev, J.C. Wells, Phys. Rev. A 57, 1849 (1998)

12. A.J. Baltz, L. McLerran, Phys. Rev. C 58, 1679 (1998)

13. A.J. Baltz, F. Gelis, L. McLerran, A. Peshier, Nucl. Phys. A 695, $395(2001)$

14. R.N. Lee, A.I. Milstein, Phys. Rev. A 61, 032103 (2000)

15. R.N. Lee, A.I. Milstein, Phys. Rev. A 64, 032106 (2001)

16. R.N. Lee, A.I. Milstein, J. Exp. Theor. Phys. 104, 423 (2007)

17. K. Hencken, D. Trautmann, G. Baur, Phys. Rev. A 51, 1874 (1995)

18. E. Bartos̆, S.R. Gevorkyan, E.A. Kuraev, N.N. Nikolaev, Phys. Lett. B 538, 45 (2002)

19. L.D. Landau, E.M. Lifshitz, Phys. Z. Sowjetunion 6, 244 (1934)

20. G. Racah, Nuovo Cim. 14, 93 (1937)

21. D.Yu. Ivanov, A. Schiller, V.G. Serbo, Phys. Lett. B 454, 155 (1999) 